\title{
Mis encuentros con Juan Carlos Tedesco
}

\section{( Alfredo Furlán y Julio U. Ríos}

\begin{abstract}
Resumen
Siguiendo una secuencia de momentos históricos de encuentros entre Alfredo Furlán y Juan Carlos Tedesco, en el presente texto se van trazando diversas etapas de las obras de Furlán en sintonía con las importantes contribuciones de Tedesco. Admirado colega y guía, a partir de haber compartido unos meses de trabajo conjunto en la Universidad Nacional de la Pampa en 1975. Partiendo de esta inicial colaboración, el escrito presenta las principales ideas y trabajos de Juan Carlos Tedesco que, a criterio de los autores, trazan un perfil de sus fundamentales aportaciones. De esta forma, se señala la importancia asignada por Tedesco a enfocar el estudio de lo educativo, situándose en el contexto histórico, social y económico en los que acontece la educación. Al mismo tiempo que se advierte la necesaria formulación de un proyecto educativo fundamentado en un proyecto político que oriente el sentido de la educación.
\end{abstract}

\section{My encounters with Juan Carlos Tedesco}

\begin{abstract}
Following a sequence of historical moments of encounters between Alfredo Furlán and Juan Carlos Tedesco, the present text traces several stages of the works of Furlán in tune with the important contributions of Tedesco. An admired colleague and guide, since the few months working together at the Universidad Nacional de la Pampa in 1975. Based on this initial collaboration, the paper presents concurrently different works and ideas of Juan Carlos Tedesco that, at the discretion of the authors, trace a profile of his main contributions. In this way, it is pointed out the importance assigned by Tedesco to focus the study of the educational, placing in the historical, social and economic context in which the education occurs. At the same time, it advises the necessary formulation of an educational project based on a political project that guides the meaning of the education.
\end{abstract}

\section{Palabras-claves:}

educación, proyecto, déficit de sentido, gestión.

\section{Keywords}

education, project, decrease of meaning, management. 


\section{Encuentro con Tedesco. Argentina, 1975}

Corría el año 1975 en Argentina en pleno gobierno de ultraderecha de Isabel Perón, cuyas políticas para con las universidades eran nombrar interventores de ideología fascista que corrían de manera inmisericorde a los profesores del sector crítico. Sin embargo, las intervenciones no eran simultáneas. Se produjo entonces una migración desde los lugares intervenidos hacia las universidades que todavía no lo habían sido. Fue así como arribamos a la Universidad Nacional de la Pampa, que era uno de los últimos lugares que acogían a algunos de los profesores dispuestos a migrar.

Nosotros, mi esposa Graciela y yo, veníamos de la Universidad Nacional de Río Cuarto y de la Universidad Nacional de Córdoba (en donde nos formamos y habíamos iniciado nuestro trabajo universitario habiendo ganado los correspondientes concursos). Nos encontramos en la Universidad Nacional de La Pampa con otros académicos migrantes: Azucena Rodríguez Ousset, que como nosotros vivía en Santa Rosa, la capital de la provincia y a Justa Ezpeleta que viajaba quincenalmente a General Pico (la segunda ciudad de la provincia donde funcionaba la carrera de Ciencias de la Educación). Allí nos encontramos con otros pedagogos que escapaban de la intervención de la Universidad Nacional del Comahue: Juan Carlos Geneyro, que había elegido como residencia General Pico y Juan Carlos Tedesco, que radicó en Santa Rosa.

Juan Carlos Tedesco era sin duda, a pesar de su juventud (tendría alrededor de 31 años), el más prestigioso pedagogo con una producción escrita de gran aceptación. Su libro Educación y Sociedad en la Argentina 1880-1945, publicado en 1972, se había convertido en un material sumamente requerido. Además, dirigía la Revista de Ciencias de la Educación, que difundía trabajos de los pedagogos críticos de todo el país y cuya publicación era esperada con ahínco por los sectores más activos vinculados a las carreras de Ciencias de la Educación.

Fue un honor trabajar en un equipo integrado por Juan Carlos Tedesco, Azucena Rodríguez Ousset, Adriana Culzoni, y el que subscribe en el área de Pedagogía Universitaria, bajo la dirección de Tedesco, nombrado por el rector como Secretario Académico. En esos meses él dictó un curso en Santa Rosa para los alumnos del profesorado con gran éxito y lleno total. Se movía como pez en el agua con temas de teorías de la educación y de historia de la educación argentina.

En una época de intervenciones era lógico pensar que permaneceríamos poco tiempo en la universidad, por lo que nadie se atrevía a elaborar planes a futuro. Por ello, desde la Pedagogía Universitaria nos encargamos de dictar unos cursos de didáctica en la Facultad de Ciencias Económicas. En todo momento, Juan Carlos se encargó de brindarnos la cobertura necesaria para desarrollar un trabajo académico que planteaba resistencias a las crudas interpelaciones intervencionistas.

La actividad académica de Juan Carlos siempre fue incesante y se caracterizaba por una mirada analítica que plasmaba con gran claridad los entramados subyacentes a la relación entre la educación y la sociedad. Las obras de esa época dan cuenta de su capacidad para analizar profundamente la educación, entrelazando rigurosamente diversos enfoques disciplinarios (históricos, económicos, sociológicos, políticos, etcétera). Una cualidad que posteriormente aportó brillantes escritos sobre la realidad de América Latina, en donde los diagnósticos se acompañaron siempre de propuestas fundamentadas en una confianza hacia los actores de los procesos educativos (Tedesco, 1995, 2012).

Juan Carlos también era un excelente deportista. Por las mañanas, temprano, se iba a jugar al frontón a unas canchas que estaban desocupadas. Al menos a esas horas. Como iba solo, tuvo a bien invitarme a jugar con él a pesar de que lo más parecido al frontón 
que yo había jugado era el ping-pong (tenis de mesa). Es decir, nada que ver. Cuando comprobó mi absoluta torpeza con la paleta y mi errático desplazamiento por la enorme cancha resolvió enseñarme, cosa que fue un bonito gesto de su parte, coherente con su inspirada confianza en la capacidad de aprendizaje de los seres humanos (Tedesco, 1995). El problema fue el corto tiempo que dispusimos para tal cometido.

En poco tiempo llegó la intervención a la Universidad. Las semanas iban pasando y no nos corrían. Por supuesto, Tedesco perdió la secretaría académica quedando como profesor. Pero al fin llegó una medida mucho más terrible. Nos buscaba la policía, allanando las casas. Cuando eso ocurrió, yo por casualidad había ido a dar clases a General Pico y regresé más tarde que habitualmente. Para ese momento la casa ya había sido allanada, por lo que fui a la casa de Tedesco para avisarle que estaba movilizada la policía. Esa fue la última vez que lo vi en Santa Rosa de La Pampa.

\section{Los encuentros posteriores. La gestión, 1991}

Como quince años después, en una ocasión que vino a México (aproximadamente por 1990), siendo director de la Oficina Regional de Educación para América Latina y el Caribe (OREALC), sostuve una conversación con Juan Carlos Tedesco en la que le planteé mi convicción de que se avecinaba una gran difusión de la noción de gestión, como management, impulsada desde los Estados Unidos en sus intentos de venderle al mundo sus modelos institucionales. La convicción se basaba en que luego de haber divulgado la problemática curricular, era previsible que se continuara con la de la gestión, porque las metodologías curriculares siempre se aplicaban a nivel de las unidades escolares, no de los sistemas educativos, como era la práctica mayoritaria en los países latinoamericanos. Se mostró de acuerdo y me recomendó que organizara un evento internacional sobre el tema. Para lo cual unimos fuerzas con Justa Ezpeleta, del Departamento de Investigaciones Educativas (DIE) del Centro de Investigación y de Estudios Avanzados (CINVESTAV), y Patricia Ducoing, del Colegio de Pedagogía de la Universidad Nacional Autónoma de México (UNAM). A partir de estos esfuerzos realizamos el seminario internacional La gestión pedagógica de los planteles escolares: prácticas, problemas y perspectivas analíticas. $\mathrm{Al}$ año del evento, se publicó en Chile $L a$ gestión pedagógica de la escuela, que reunió la mayor parte de los trabajos presentados en el seminario. Justa Ezpeleta y yo somos los compiladores del libro que se distribuyó por toda América Latina y el Caribe. En este, Juan Carlos escribió un magnífico capítulo llamado "Estrategias de desarrollo y educación: el desafío de la gestión pública", donde postula unos planteamientos acerca de la situación política y económica en la cual se inserta la cuestión de la gestión. Dice, por ejemplo:

Permitir mayores grados de control de los procesos y de los resultados por parte de los destinatarios (padres, organizaciones de la comunidad, docentes, etc.) e implementar eficientes sistemas de información pública, constituyen elementos centrales para introducir una dinámica diferente en la gestión de políticas educativas públicas. Pero la aplicación de estos criterios supone modificar la estructura de la administración educacional, pasando cuotas cada vez mayores de actividades al sistema de administración por programas, a través de contratos de servicios, en lugar del sistema regular de administración. (Tedesco, 1992: 40).

El estilo un tanto abstracto es debido, creo, a su papel de funcionario de la Organización de las Naciones Unidas para la Educación, la Ciencia y la Cultura (Unesco) que, según me dijo tiempo después, se veía obligado a adoptar cuando hablaba oficialmente. No obstante, dentro de estas concesiones institucionales se formulaban las proposiciones que comenzaron a identificar en la crisis de los años noventa un evento que no era 
simplemente de tipo coyuntural, sino que representaba "el agotamiento del patrón de desarrollo que la región había mantenido hasta entonces" (Tedesco, 2012: 84). Al respecto, como Juan Carlos reconoció en años recientes: "si bien hoy disponemos de evidencias relativamente claras acerca de las características centrales de este proceso de cambio, en aquellos momentos iniciales solo era posible postular hipótesis, en gran medida cargadas de intenciones y deseos de sus propios autores" (2012: 83).

Lo planteado por Tedesco es una advertencia de las transformaciones que se gestaban y que tienen hoy día explícitas repercusiones en la relación entre educación, mercado y ciudadanía. Tal situación fue presentada por él en los siguientes términos reveladores de lo que se configuraba:

\begin{abstract}
El mundo del trabajo se ha complejizado en forma notoria y la actividad productiva se articula cada vez más estrechamente con la actividad intelectual: por un lado, la producción requiere mayores niveles de logro en determinadas capacidades humanas como la creatividad, la inteligencia, la selección de información; por el otro, la actividad productiva no solo consume conocimientos, sino que los produce. Por esta razón, actualmente una educación desligada del mundo del trabajo no solo es regresiva desde el punto de vista económico, sino empobrecedora desde la perspectiva del desarrollo integral de la personalidad individual. (Tedesco, 1992: 23)
\end{abstract}

Ante la crisis el diagnóstico, pero también la propuesta concreta de reflexionar sobre el significativo papel que la educación puede tener en un incierto escenario social (Tedesco, 1995). Tal es la permanente perspectiva que Juan Carlos conservó en sus textos. En los que apremió a tomar posiciones, a delimitar las acciones a seguir y asumir la duda como cualidad necesaria que evita caer en dogmatismos que solo entorpecen nuestra capacidad para proyectarnos hacia el futuro con confianza (Tedesco, 1995).

\title{
3. La disciplina y la violencia, 1998
}

Unos ocho años más tarde le compartí a Juan Carlos Tedesco mi interés por estudiar el problema de la disciplina escolar y la violencia. Me propuso que, si yo podía armar un dossier sobre el tema, él lo publicaría en la revista Perspectivas del Buró Internacional de la Educación que dirigía en ese momento. El dossier se publicó en 1998 y fue traducido, como se hacía normalmente con la revista, a cinco idiomas.

Durante esa época, sus escritos dan cuenta de la profunda síntesis que magistralmente articuló en torno a la relación entre la educación y la sociedad. La crisis que desde años atrás concebía como distinta de los cíclicos periodos de ajuste del capitalismo, se desplegaba hacia finales de los años noventa e inicios del siglo XXI como la nueva forma del modelo económico y social. Entre el pesimismo o el optimismo en torno a lo que se desarrollaba, Tedesco (1995) escogió un voluntarismo consciente que deposita su confianza en la capacidad de los seres humanos para aprender. En este sentido, el nuevo pacto educativo al que hace mención es un llamado a los actores sociales para forjar acuerdos y consensos a través de la concertación. Lo que:

Supone, en consecuencia, reconocer al otro y negociar formas de trabajo común. La concertación no elimina el conflicto, ni las tensiones, ni las diferencias. No significa uniformidad. [...] Pero crea un mecanismo a través del cual esos conflictos y tensiones son resueltos mediante el diálogo y los acuerdos para la acción. (Tedesco, 1995: 185)

Así, los planteamientos de Juan Carlos se fueron enfocando a la construcción de propuestas frente a los cambios ya en desarrollo. Labor que realizó incansablemente desde 
la Unesco. Por esos años en que se difundió el dossier, Juan Carlos regresó a Buenos Aires como director de la sucursal del Instituto Internacional de Planeamiento de la Educación (IIPE) que, junto a la OREALC, son las sucursales de la Unesco en América Latina. Lo visité en varias ocasiones. Los encuentros siempre fueron muy cordiales.

En una ocasión me obsequió una colección de la Revista de Ciencias de la Educación que él había fundado y dirigido a fines de los sesenta y principio de los setenta. La cual atesoro con mucho cariño, ya que esta fue un suceso extraordinario por la perspectiva amplia de su contenido; atribuible a la entusiasta y firme conducción de Juan Carlos. En ella se podían encontrar textos de Jean Claude Filloux, Pablo Latapí, Pedro Lafourcade, Telma Barreiro, Rodolfo Bohoslavsky, Clotilde Yapur, Alicia Camillioni, R. Cunha, Ivan Illich, J. C. Passeron y muchos más. Entre ellos, muchos argentinos que encontraron en la revista una magnífica oportunidad para dar a conocer sus estudios. Al analizar la precariedad de los medios disponibles a comienzo de los setenta es notorio que su publicación fue resultado de un enorme esfuerzo. Particularmente, la diversidad de las temáticas abordadas y los enfoques incluidos en la revista han hecho que esta permanezca vigente. En este sentido, su lectura continúa siendo ampliamente recomendada.

Con una inocultable emoción escuché varias conferencias suyas que dio en los últimos años (¡oh magia del YouTube!) y percibo que en las últimas de ellas es mucho más explícito en plantear la dimensión política transformadora: falta definir un proyecto de país, para que se pueda lograr una sociedad más justa que le dé sentido a la educación.

De esta forma, las transformaciones que se producían en la familia, el Estado, la tecnología, el mercado y la escuela serán analizadas por Tedesco señalando especialmente el aumento de la desigualdad en América Latina. Advirtiendo además de los peligros de un individualismo que corre el riesgo de ensimismarse en la libertad individual, olvidándose del compromiso social que tiene cada individuo (Tedesco, 2000). Un riesgoso panorama donde aquellos que se mantengan fuera de las nuevas formas de producción y transmisión de la información y el conocimiento serían los más vulnerables ante el desvanecimiento de los tradicionales acuerdos sociales.

\section{El problema del sentido, 2014}

Hace ya tres años, organizamos en Iztacala un simposio denominado El sentido de la educación y la escuela, al cual lo invitamos. Pero no pudo venir porque en los mismos días en que habíamos programado el evento, había recibido la invitación de Girona, Cataluña (España), donde le entregarían el doctorado honoris causa. El reconocimiento hecho durante esta época a Juan Carlos Tedesco se enmarca en el profundo alcance logrado por su obra. En la que, con excepcional claridad y rigurosidad, nos concedió un brillante análisis de las implicaciones aparejadas al nuevo capitalismo (Tedesco, 2012).

Como Tedesco (1995) mostró, este nuevo modelo económico y social se caracteriza especialmente por la pérdida de sentido, es decir, la ausencia de finalidades a las cuales aspirar. Una pérdida que se da a la par del desmoronamiento de la certidumbre que acompañaba a las tradicionales instituciones sociales, cuyas funciones se ven debilitadas en el marco de las nuevas políticas neoliberales. Lo que particularmente se ha traducido actualmente en América Latina en un cuestionamiento de la educación y la escuela (Tedesco, 2012).

Mientras que las pasadas certezas generan desconfianza al ser consideradas caducas, el futuro es inseguro debido a que las bases que anteriormente lo cimentaban se han desdibujado. La única opción que queda es el presente, el aquí y ahora. Como precisó 
Tedesco, "la profundidad de los cambios, su rapidez y la incertidumbre acerca de su dirección, generan una fuerte concentración en el presente, fenómeno identificado como el más característico de la cultura de este nuevo capitalismo" (2012: 132). Aspecto que en el caso de la educación tiene efectos:

Particularmente nocivos pues se supone que la educación está destinada a transmitir el patrimonio cultural y a preparar para el futuro. Si lo pasado está asociado con lo obsoleto y el futuro es incierto, la concentración en el presente deja a los educadores sin uno de los fundamentos básicos de su tarea. En el caso particular de los docentes, la ausencia de información o de comprensión acerca del sentido del proceso global de cambio provoca un fenómeno según el cual la transformación está asociada fundamentalmente a la idea de perdida y a sentimientos de inseguridad y de incertidumbre sobre el pasado. (2012: 106)

Pero la teoría del déficit de sentido no es solo un sobresaliente marco de referencia que muestra los peligrosos destinos a los que nos podríamos acercar de no tomar las decisiones necesarias para cubrir la ausencia de sentido. Es también la advertencia desde la que Tedesco dirigió sus esfuerzos hacia una adhesión a la justicia social, concepto central de un sentido socialmente compartido (Tedesco, 2012). El logro de la justicia social dota al momento presente de una finalidad, una aspiración que orienta los esfuerzos de una educación que promueve "altos niveles de adhesión a la justicia social como rasgo cultural compartido y, a su vez, exige la existencia de esa adhesión para legitimar su tarea socializadora" (2012: 142).

\section{El legado}

Los encuentros con Juan Carlos Tedesco son un encuentro simultáneo con su vida y obra. Un legado cuya trascendencia difícilmente podría ser abarcada en su totalidad dentro del presente trabajo. De ahí que referirse a sus aportes en las próximas líneas no es tanto una síntesis puntual de los mismos, como una serie de orientaciones que permitan guiar de forma general la reflexión sobre los temas que tan rigurosamente analizó en una portentosa cantidad de escritos. En los que a semejanza del maestro artesano:

Comprende la importancia del esbozo al comienzo de su trabajo, cuando no tiene un conocimiento acabado de los detalles del objeto que desea construir, asigna valor a la contingencia, evita el perfeccionismo y aprende cuando es el momento de detenerse. Más trabajo puede empeorar las cosas. (Tedesco, 2012: 169)

Con gran dominio de su oficio, en sus textos Juan Carlos Tedesco realizaba un acompañamiento a quienes nos adentrábamos a sus ideas. Como buen maestro, "supo transmitir emociones con respecto al trabajo, fundamentalmente la pasión por la tarea bien realizada, la preocupación por el aprendizaje [...] y el valor del conocimiento" (2012: 168). Una tarea que desarrolló a partir de una rigurosa fundamentación teórica en la que se contaba con la lectura de los más lúcidos aportes de los intelectuales de la época: Pierre Rosanvallon, Richard Sennett, Alain Touraine, entre otros.

En algún momento, Juan Carlos caracterizó a uno de sus libros como "un texto ecléctico en más de un sentido" (1995: 10). Referencia a la tendencia que tuvo siempre de combinar con maestría diferentes enfoques y posturas, rechazando siempre la trampa de opciones dicotómicas que perjudicialmente muestran al mundo dividido en dos opciones. En este sentido, mantuvo un constante rechazo a toda forma de dogmatismo (Tedesco, 2012). Esto se tradujo en una apertura para no aislar el análisis del fenómeno educativo del estudio de las tendencias que existen a nivel mundial y a nivel regional. 
Tedesco tomó posiciones claras sobre la relación entre la educación y la sociedad, pero también incorporó la duda y el interrogatorio como aspectos fundamentales de una reflexión que no se queda con la inmediatez de lo visible. Enseñándonos de esta forma a tratar de historizar el desarrollo de las coordenadas que caracterizan a las prácticas educativas, cada vez que nos referimos a ellas. Se trata de desmitificar el pasado de las miradas nostálgicas y conservadoras (Tedesco, 2015). Desde este planteamiento, se entiende mejor el porqué de la orientación dada por Juan Carlos para analizar las políticas educativas en el contexto en que se producen, moderando la crítica a una estimación de lo posible.

Abordar la educación desde enfoques diversos, asumiendo compromisos con posturas abiertas al cuestionamiento. Esa podría ser quizá una insuficiente síntesis del congruente quehacer de Juan Carlos, quien asumió siempre muy en serio todas las actividades profesionales que encaró (llegó incluso a definirse, por ejemplo, como un hombre de la Unesco, en estricto sentido de la palabra), al mismo tiempo que supo tomar una prudente distancia de los papeles oficiales usando un sentido del humor que afloraba en los momentos más insospechados. Referente de necesaria consulta para entender el momento actual de la educación y así proyectarse hacia el futuro (Tedesco, 1995). Juan Carlos Tedesco es, desde años atrás, un clásico de la educación. 


\section{Q Referencias bibliográficas}

» Tedesco, J. C. (1986). Educación y Sociedad en la Argentina 1880-1945. Buenos Aires, Solar.

»_. (1992). Estrategias de desarrollo y educación: el desafío de la gestión pública. En Ezpeleta, J. y Furlán, A. (comps.), La gestión pedagógica de la escuela, pp. 21-44. Santiago, Unesco/OREALC.

»_._. (1995). El nuevo pacto educativo. Educación, competitividad y ciudadanía en la sociedad moderna. España, Anaya.

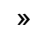
. (2000). Educar en la sociedad del conocimiento. Buenos Aires, Fondo de Cultura Económica.

»__. (2012). Educación y justicia social en América Latina. Buenos Aires, Fondo de Cultura Económica.

"_. (2015). Conclusiones. Política educativa: justicia social y responsabilidad por los resultados. En Tedesco, J. C. (comp.), La educación argentina hoy: la urgencia del largo plazo, pp. 423-443. Buenos Aires, Siglo Veintiuno.

\section{Breve currículum de los/as autores/as}

\section{Alfredo Furlán}

Doctor en Ciencias de la Educación, Université René Descartes (Paris V). Profesor titular, Facultad de Estudios Superiores-Iztacala, Universidad Nacional Autónoma de México. Correo electrónico: furlan@unam.mx

\section{Julio U. Ríos}

Maestro en Pedagogía, Facultad de Filosofía y Letras, Universidad Nacional Autónoma de México. Asistente de investigación, Universidad Nacional Autónoma de México (UNAM). Correo electrónico: ripju_cnsl@hotmail.com 